\title{
Molecular complexes of phenols with DDQ
}

\author{
T VINOD KUMAR, T VEERAIAH and G VENKATESHWARLU* \\ Department of Chemistry, Nizam College, Osmania University, \\ Hyderabad 500 001, India
}

MS received 11 October 1999; revised 23 March 2000

\begin{abstract}
Molecular complexes of phenols with DDQ have been studied spectrophotometrically in the temperature range of $10-30^{\circ} \mathrm{C}$ in a solvent $\left(\mathrm{CHCl}_{3}\right)$ of low polarity under low donor concentrations. All the complexes exhibit one CT band each in the wavelength region where acceptor and donor do not have any absorption. The complexes are inferred to be of the $\pi-\pi$ type and have $R_{y}$ configuration in which the donor molecular orbital encompasses the substituent. The ionization potentials of the donors, the stability constants and thermodynamic parameters of the complexes have been evaluated.
\end{abstract}

Keywords. DDQ-phenols; CT complexes; configuration; stability constants.

\section{Introduction}

In continuation of our work on the donor abilities of phenols and their tendency to form charge transfer (CT) complexes with $\pi$ acceptors ${ }^{1}$, we considered it interesting to investigate the CT spectra, stabilities and thermodynamic parameters of CT complexes of phenols with 2,3-dichloro-5,6-dicyano-p-benzoquinone (DDQ). Literature survey revealed that DDQ forms CT complexes with phenols and produces a characteristic colour in methanol ${ }^{2,3}$, but the colour fades with time indicating that these complexes are unstable and soon turn into other products. It is for this reason that the characteristics of CT complexes, viz., positions of CT bands, stabilities and thermodynamic parameters have not yet been reported. Further, we noticed from literature ${ }^{4,5}$ that the CT complexes can be stabilized for a longer time in solvents of low polarity, at low temperatures and under low donor concentrations. To study this further we chose a low polar solvent, viz. $\mathrm{CHCl}_{3}$, and recorded the spectra of these complexes at low temperatures using low concentrations of phenols. The results are reported here.

\section{Experimental}

The commercial sample of DDQ obtained from Aldrich was repeatedly recrystallized from benzene-chloroform (2:1) mixture (m.p. $213-214^{\circ} \mathrm{C}$ ). Phenols (BDH-Analar) were purified by the methods available in literature ${ }^{6}$. Spectrograde chloroform (BDH) was used without further purification.

The UV-vis absorption spectra (figure 1) of the complexes were recorded on a Shimadzu-240 double beam spectrophotometer on fast scan speed in the temperature

*For correspondence 


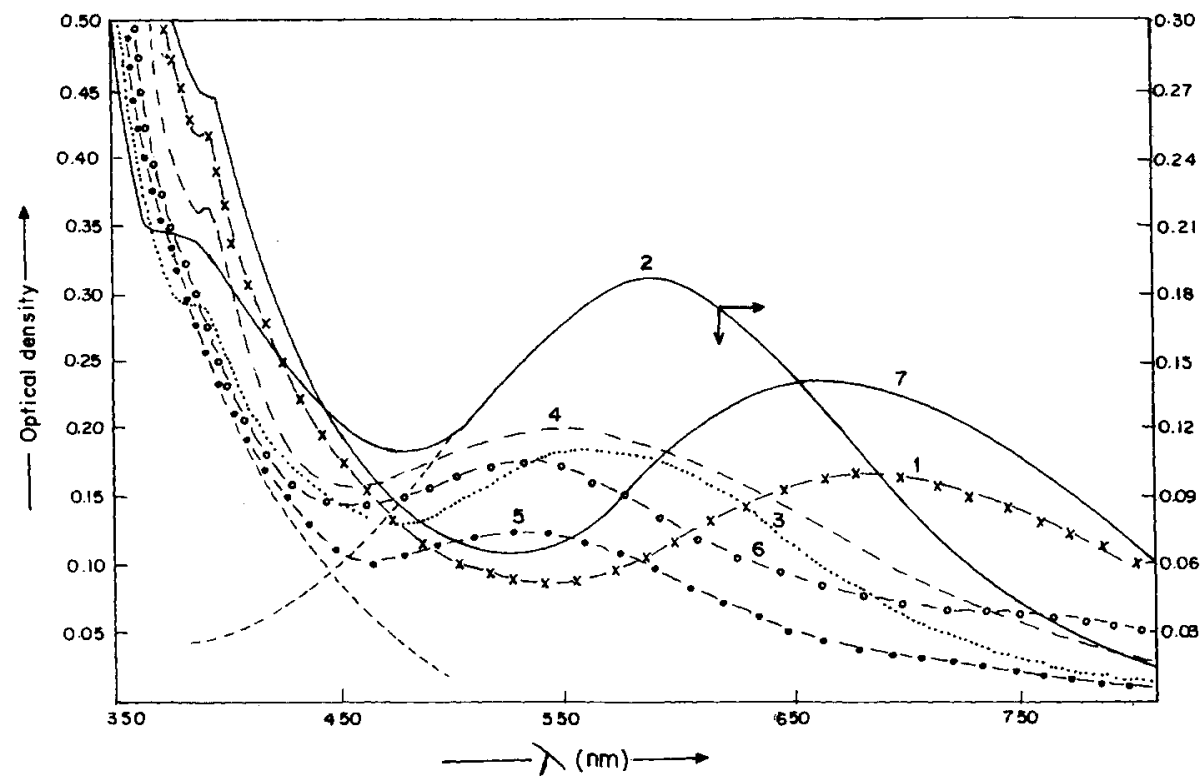

Figure 1. Charge transfer spectra of DDQ complexes with phenols and naphthols (1, $\alpha$-naphthol; 2, $\beta$-naphthol; 3, $p$-methoxy phenol; 4, $p$-cresol; $5, o$-cresol; $6 . m$ cresol; 7,p-chloro phenol; 8, $p$-bromo phenol and 9, phenol.)

range $10-30^{\circ} \mathrm{C}$ at $5^{\circ} \mathrm{C}$ intervals with an accuracy of $\pm 0 \cdot 1{ }^{\circ} \mathrm{C}$ using a matched pair of quartz-stoppered cells of $10 \mathrm{~mm}$ path length. The studies at different temperatures were made in a thermostatted sample compartment by circulating water around the cuvettes. Stock solutions of donor and acceptors were prepared and were mixed just before recording the spectra. The concentration of DDQ varied between $1 \times 10^{-4}$ and $5 \times 10^{-4} \mathrm{M}$ while that of donors between 0.005 and $0.15 \mathrm{M}$. Absorption bands due to the acceptor or donor alone have fallen to the base line much before the wavelength of CT absorption. However, the lower wavelength side of the CT band is further complicated by other absorptions, probably due to the complexed donor ${ }^{7}$. The complicated CT bands are analysed using the relation given by Briegleb and Czekalla ${ }^{8}$,

$$
v_{h}-v_{l} / 2\left(v_{m}-v_{l}\right)=1 \cdot 2,
$$

where $v_{h}$ and $v_{l}$ refer to the frequency at half the maximum intensity of the high and low frequency sides of the peak located at $v_{m}$. The formation constants $(K)$ of the complexes were determined by using both Benesi-Hildebrand ${ }^{9}$ and Rose-Drago ${ }^{10}$ equations,

$$
\begin{aligned}
& {\left[A_{0}\right] / A=1 / K\left[D_{0}\right] \varepsilon+1 / \varepsilon,} \\
& K^{-1}=A / \varepsilon-\left(\left[A_{0}\right]+\left[D_{0}\right]\right)+\left[A_{0}\right]\left[D_{0}\right] \varepsilon / A,
\end{aligned}
$$


where $K$ is the stability constant, $A$ is absorbance and $\varepsilon$ is molar extinction coefficient of the complex. $\left[A_{0}\right]$ and $\left[D_{0}\right]$ are initial concentrations of acceptor and donor respectively.

Stability constants are obtained from the ratio of intercept to slope and $\varepsilon$ from inverse intercept of Benesi-Hildebrand plots (figure 2). They are also determined from the intersection points of the Rose-Drago plots. The values obtained by both the methods agreed well with each other. Thermodynamic parameters, viz. $\Delta H$ and $\Delta S$, have been determined from the temperature variation of stability constants using van't Hoff's method and are reported in table 1 together with $\Delta G$ at $25^{\circ} \mathrm{C}$. The stoichiometry of each of the complexes was determined by Job's continuous variation method and is found to be 1:1 (figure 3).

\section{Results and discussion}

Colourless solutions of DDQ and phenols in chloroform turned blue immediately after mixing. The colour changes are attributed to the formation of CT complexes between the DDQ and the phenols. All the complexes exhibit one CT band each in the range 525 to $680 \mathrm{~nm}$. Appearance of CT bands is attributed to the excitation of electron from the highest occupied molecular orbital (HOMO) of donor to the lowest unoccupied molecular

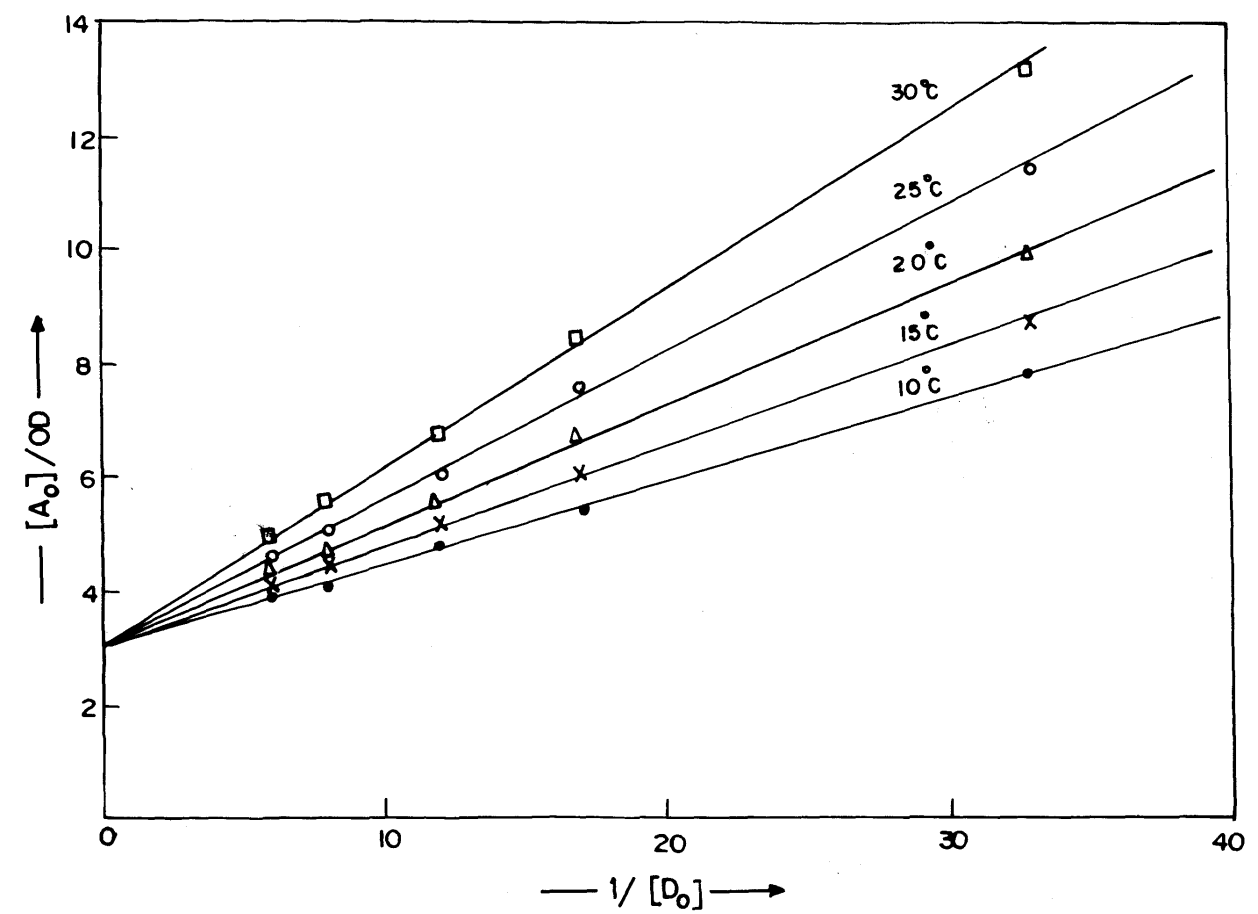

Figure 2. Benesi-Hildebrand plots of CT complexes of DDQ with phenol at different temperatures. 
Table 1. Charge transfer spectra, stability constants $(K)$ and thermodynamic parameters of DDQ-phenol complexes.

$\Delta H$ and $\Delta G$ in k cal mol${ }^{-1} ; \Delta S$ in cal $\mathrm{deg}^{-1} \mathrm{~mol}^{-1} ; \varepsilon$ in $1 \mathrm{~mol}^{-1} \mathrm{~cm}^{-1} ; \lambda$ in $\mathrm{nm}$; IP in eV; $K$ in $1 \mathrm{~mol}^{-1}$. The values of stability constants $(K)$ are accurate up to $\pm 0 \cdot 25$.

\begin{tabular}{|c|c|c|c|c|c|c|c|c|c|c|c|}
\hline \multirow[b]{2}{*}{ Donor } & \multirow[b]{2}{*}{$\lambda \mathrm{CT}$} & \multirow[b]{2}{*}{$\varepsilon$} & \multirow[b]{2}{*}{ IP } & \multicolumn{5}{|c|}{$K^{\circ} \mathrm{C}$ at } & \multirow[b]{2}{*}{$\Delta H$} & \multirow[b]{2}{*}{$\Delta S$} & \multirow[b]{2}{*}{$\Delta G$} \\
\hline & & & & 10 & 15 & 20 & 25 & 30 & & & \\
\hline$\alpha$-Naphthol & 735 & 4590 & $7 \cdot 64$ & 156 & 103 & 71 & 48 & 34 & $13 \cdot 0$ & $35 \cdot 9$ & $2 \cdot 31$ \\
\hline$\beta$-Naphthol & 693 & 4960 & $7 \cdot 77$ & 146 & 99 & 68 & 47 & 33 & $12 \cdot 74$ & $35 \cdot 10$ & $2 \cdot 28$ \\
\hline$p$-Methoxy phenol & 680 & 5765 & $7 \cdot 81$ & 85 & 60 & 43 & 31 & 23 & $11 \cdot 20$ & $30 \cdot 74$ & $2 \cdot 04$ \\
\hline$p$-Cresol & 595 & 4100 & $8 \cdot 14$ & 54 & 40 & 30 & 23 & 17 & $9 \cdot 62$ & $26 \cdot 06$ & 1.85 \\
\hline$o$-Cresol & 580 & 3940 & $8 \cdot 22$ & 50 & 37 & 28 & 21 & 16 & $9 \cdot 54$ & $25 \cdot 92$ & $1 \cdot 81$ \\
\hline$m$-Cresol & 570 & 3960 & $8 \cdot 26$ & 33 & 26 & 20 & 16 & 13 & $8 \cdot 20$ & $22 \cdot 02$ & 1.64 \\
\hline p-Chlorophenol & 560 & 3050 & $8 \cdot 32$ & 23 & 19 & 15 & 12 & 10 & $7 \cdot 10$ & $18 \cdot 83$ & 1.49 \\
\hline p-Bromophenol & 550 & 2950 & $8 \cdot 37$ & 20 & 16 & 13 & 11 & 9 & $6 \cdot 95$ & $18 \cdot 59$ & 1.41 \\
\hline Phenol & 537 & 2400 & $8 \cdot 44$ & 12 & 10 & $8 \cdot 7$ & $7 \cdot 5$ & $6 \cdot 3$ & $5 \cdot 68$ & $15 \cdot 08$ & $1 \cdot 18$ \\
\hline
\end{tabular}

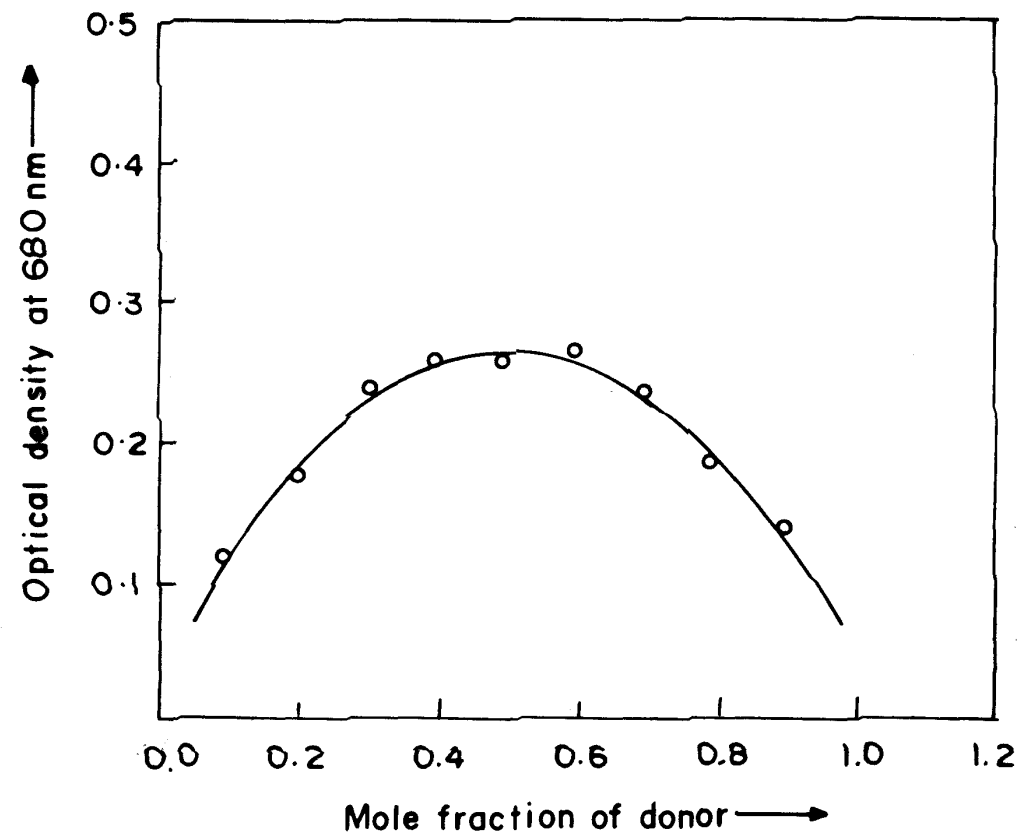

Figure 3. Job's continuous variation plot of DDQ with $p$-methoxyphenol $\left[D_{0}\right]=\left[A_{0}\right]=2 \cdot 0 \times 10^{-3} \mathrm{M}$.

orbital (LUMO) of the acceptor. Here it is interesting to note that phenols exhibit two CT bands with TCNE. The higher and lower wavelength bands were attributed to excitation of electron from ultimate $\left(\psi_{s}\right)$ and penultimate $\left(\psi_{a s}\right)$ molecular orbitals of the donor thus 
forming complexes with two different configurations viz., $R_{x}$ and $R_{y}$. In order to determine the configuration of the phenols-DDQ complexes, the $v_{\mathrm{CT}}$ of phenols-DDQ are plotted against those of $R_{x}$ and $R_{y}$ configurational complexes of phenols-TCNE. A linear relationship is observed between the $v_{\mathrm{CT}}$ of phenols-DDQ and the $v_{\mathrm{CT}}$ of $R_{y}$ configuration of phenols-TCNE while that of $R_{x}$ configuration exhibited a scatter. A linear relation is observed only when the same orbital of donors is involved in the formation of complexes with two different acceptors. It is therefore inferred that in phenol-DDQ complexes also the $\psi_{s}$ orbital acts at a donor level and forms complexes with $R_{y}$ configuration in which the energy of the CT transition is affected by the substituents as this level encompasses the substituent ${ }^{1}$ (figure 4). The absence of $R_{x}$ configurational complex implies that the $R_{y}$ configuration is more stable than the $R_{x}$ configuration. This is reasonable because in $R_{y}$ configuration the $\mathrm{C}=\mathrm{O}$ of $\mathrm{DDQ}$ and the $\mathrm{O}-\mathrm{H}$ of phenol lie parallel and may form hydrogen bonding which significantly favours this configuration over the other.

\subsection{Effect of substitutents on CT bands}

The position of CT band of phenol-DDQ $(530 \mathrm{~nm})$ is shifted to longer wavelengths by the substituents present in the phenol ring and the shifts are in the following order.

$\alpha$-naphthol $>\beta$-naphthol $>p$-methoxy phenol $>p$-cresol $>o$-cresol $>m$-cresol $>p$ chloro phenol > $p$-bromo phenol > phenol.

The effect of substituents on the position of the CT band is attributed to the interaction of substituents with the $\pi$ orbitals of the benzene ring of phenol. The conjugative interaction of naphthalene, the mesomeric effect of $\mathrm{OCH}_{3}$, hyperconjugative effect of $\mathrm{CH}_{3}$ and inductive effect of halogen groups all perturb the resonance in the benzene ring and boost the energy of the donor $\psi_{s}$ level and bring it closer to the LUMO of DDQ and thus cause a bathochromic shift in the CT band.

\subsection{Ionisation potentials of the donor}

The ionisation potentials of the phenols were calculated from the following equation ${ }^{11}$,

$$
h v_{\mathrm{CT}}=0.77 I_{\mathrm{D}}-4 \cdot 19,
$$

and are reported in table 1.
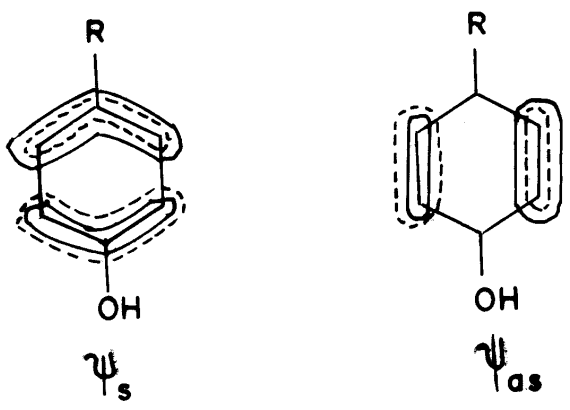

Figure 4. The $\psi_{\mathrm{s}}$ and $\psi_{\text {as }}$ molecular orbitals of benzene. 
The values of ionization potentials obtained in this study are comparable to those obtained from the CT spectra of phenols-TCNE complexes and the photoionization method. They are however lower compared to those obtained from the electron impact method. The ionisation potential of phenol is very close to that obtained from photoelectron spectroscopy for the electrons in $\pi$-HOMO and far lower than those of electrons in other orbitals ${ }^{12,13}$. This supports our assumption that electron donation takes place from the $\psi_{s}$ molecular orbital.

\subsection{Formation constants and thermodynamic parameters}

The stabilities of complexes increase with increasing electron-releasing ability of the substitutents in the benzene ring and are in the sequence:

$\alpha$-naphthol $>\beta$-naphthol $>p$-methoxy phenol $>p$-cresol $>o$-cresol $>m$-cresol $>$ phenol $>p$-chloro phenol $>p$-bromo phenol.

The formation constants of $\alpha$-naphthol and $\beta$-naphthol complexes are greater than that of phenol complex and are in the order: $\alpha$-naphthol $>\beta$-naphthol $>$ phenol. The logarithmic functions of formation constants bear a linear relationship with the Hammett $\sigma_{p}$ constants (figure 2).

It is interesting to note that the stability constants of the phenols-DDQ complexes are found to be greater than those of the corresponding phenols-TCNE complexes ${ }^{1}$ and they are also greater than the correspondingly substituted benzenes-DDQ complexes, while the stabilities of $\alpha$-naphthol and $\beta$-naphthol-DDQ complexes are greater than that of naphthalene-DDQ ${ }^{14}$. The higher stability of DDQ complexes over those of TCNE may be due to the greater electron affinity of DDQ over the TCNE. The greater stability of the phenols-DDQ over the benzenes-DDQ may be due to greater polarity of phenols over benzenes as discussed elsewhere ${ }^{1}$.

Thermodynamic parameters viz. $\Delta H, \Delta S$ and $\Delta G$ of the complexes have been determined from the variation of $K$ with temperature (table 1). Plots of $\log K$ vs $1 / T$ give straight lines, from the slope and intercept of which $\Delta H$ and $\Delta S$ can be evaluated. The $\Delta G$ values are calculated from the equation

$$
\Delta G=\Delta H-T \Delta S,
$$

and are reported at $25^{\circ} \mathrm{C}$. The $\Delta H$ values are found to be about $10 \mathrm{~K} \mathrm{cal} \mathrm{mol}^{-1}$ a characteristic feature of CT complexes. The negative enthalpies show that the complex formation is spontaneous, while negative entropy indicates a decrease in the degree of freedom of the components upon complexation. The plot of $\Delta H$ vs $\Delta S$ which is a straight line indicates that the complex formation is sterically unhindered. This favours a sandwich structure for the complexes in which the $\pi$ orbital of donor and $\pi^{*}$ orbital of acceptor can have greater possible overlap ${ }^{15}$.

\section{Acknowledgements}

We wish to thank Dr D Malleshwar and Dr B Gopala Rao, Nizam College, Hyderabad. We also thank B Subrahmanyam for helpful discussion and for correcting the manuscript. TVK thanks B Yadagiri Reddy of SAP College for his encouragement. 


\section{References}

1. Shanta M, Suresh T and Venkateshwarlu G 1998 Indian J. Chem. A37 1119

2. Becker H D 1965 J. Org. Chem. 30982

3. Hewgill F R and Howie G B 1978 Aust. J. Chem. 31907

4. Rappoport Z 1963 J. Chem. Soc. 4498

5. Rappoport Z and Horowitz A 1964 J. Chem. Soc. 1348

6. Vogel A I 1978 A text book of practical organic chemistry 4th edn (London: ELBS) p 739

7. Fornasiero D and Grieser F 1990 J. Chem. Soc., Faraday Trans. 86296

8. Briegleb G and Czekalla J 1960 Physik. Z.Chem. 2437

9. Benesi H A and Hildebrand J H 1949 J. Am. Chem. Soc. 712703

10. Rose N and Drago R S 1951 J. Am. Chem. Soc. 816138

11. Canselier J P, Domenech S, Stankovsky S and Gautier S 1982 Can. J. Chem. 60862

12. Agren H and Bagus P S 1985 J. Am. Chem. Soc. 107134

13. Debies T P and Rabalais J W 1972 J. Electron Spectrosc. 1355

14. Foster R 1969 Organic charge transfer complexes (London: Academic Press) p 202

15. Mulliken R S 1954 J. Chim. Phys. 51341 